\title{
REVISÃO DA LITERATURA DO MODELO DE ESTOQUE: LOTE ECONÔMICO DE COMPRA - LEC
}

\author{
Kelly Pereira Pepino \\ Universidade Federal do Rio Grande - FURG \\ Av. Itália, km 8, bairro Carreiros, Rio Grande, RS, Brasil \\ kellypepino@hotmail.com \\ André Andrade Longaray \\ Universidade Federal do Rio Grande - FURG \\ Av. Itália, km 8, bairro Carreiros, Rio Grande, RS, Brasil \\ andrelongaray@furg.br \\ Paulo Roberto da Silva Munhoz \\ Universidade Federal do Rio Grande - FURG \\ Av. Itália, km 8, bairro Carreiros, Rio Grande, RS, Brasil \\ paulorsmunhoz@hotmail.com \\ Diogo Garcia Storino \\ Universidade Federal do Rio Grande - FURG \\ Av. Itália, km 8, bairro Carreiros, Rio Grande, RS, Brasil \\ diogo.storino@hotmail.com
}

\section{RESUMO}

A maioria dos empreendedores busca a ideia de maximizar o lucro e reduzir os custos dos seus produtos ou serviços sem prejudicar a qualidade dos mesmos, assim alcançando a um desempenho ideal no qual é de ter uma organização mais eficiente evitando perdas e otimizando seus recursos. Utilizar a racionalização da gestão de estoque é uma maneira importante para conquistar os objetivos priorizando a diminuição de custos. $\mathrm{O}$ presente trabalho tem como objetivo executar uma revisão da literatura referente ao lote econômico de compra - LEC, com a intenção de verificar se o mesmo melhora as condições de custos. O estudo é realizado através da revisão bibliográfica dos artigos encontrados nas bases de dados Proquest, Scopus, Science Direct e Web of Science sem nenhuma restrição, as quais são coletados os dados e analisados.

Palavra-chave: Lote Econômico de Compras, LEC, Controle de Inventário, Estruturas de custos, Dimensionamento de lote, Gestão de Pedido, Otimização da Quantidade do Pedido.

\begin{abstract}
Most entrepreneurs pursue the idea of maximizing profit and reducing the cost of their products or services without compromising their quality, thus achieving optimal performance in which to have a more efficient organization avoiding losses and optimizing its resources. Utilizing inventory management rationalization is an important way to achieve goals by prioritizing cost savings. The present work aims to perform a literature review regarding the economic purchase lot - LEC, with the intention of verifying if it improves the
\end{abstract}


cost conditions. The study is conducted through the literature review of articles found in the databases Proquest, Scopus, Science Direct and Web of Science without any restrictions, which are collected and analyzed.

Keywords: Economic Order Quantity, EOQ, Inventory Control, Cost Structures, Lot-sizing, Order Management, Optimization of the order quantity.

\section{Como Citar:}

PEPINO, Kelly Pereira et al. Revisão da literatura do modelo de estoque: lote econômico de compra - lec. In: SIMPÓSIO DE PESQUISA OPERACIONAL E LOGÍSTICA DA MARINHA, 19., 2019, Rio de Janeiro, RJ. Anais [...]. Rio de Janeiro: Centro de Análises de Sistemas Navais, 2019.

\section{INTRODUÇÃO}

O investimento de um produto ou serviço prestado é a base para que se faça o preço final destes. E que a falta da eficácia na gestão pode afetar a margem de lucro e as vendas devido à incorreta cobrança de valores por não estarem conforme a realidade [13].

A gestão de estoque é uma questão essencial e é responsável por consumir grande parte do orçamento operacional de uma empresa. E que será mais eficiente uma produção quanto menor for seu estoque para produzir, analisando que o estoque não agrega valor para o produto fim [9].

Os empreendedores foram afetados pela crise econômica nos últimos tempos, e em geral a maior parte das soluções para se recuperar é adequar para alcançar uma boa gestão, incluindo a gestão de estoques [20].

Através de uma gestão adequada pode-se alcançar um equilíbrio entre os setores de vendas e produção, suprindo todas as demandas de forma continua. O que traz previsibilidades das demandas assim resultando na melhor identificação do que precisa ser otimizado para alcançar a redução de custos necessária [14].

$\mathrm{O}$ que visa à garantia da melhor disponibilidade dos produtos, tendo o menor estoque, é a gestão. E um bom planejamento de demanda é o suporte que a empresa deve buscar executar combinado com o controle do inventário e a qualidade do depósito para conquistar a eficiência do estoque. As estatísticas atuais de custo de estoque no Brasil, têm dados como 3\% do PIB - Produto Interno Bruto - nacional e cerca de $2 \%$ da receita líquida das organizações [12].

Uma situação de estoques ineficaz pode levar a falhas, ou seja, faltas que não estão esperadas de produtos nos depósitos. Como resultado dessa descontinuidade a consequência é a baixa do indicador de fill rate, medidor de pedidos atendidos. Abandono é a possível reação para aqueles consumidores os quais não são atendidos de uma forma completa que os satisfaçam, isso pode trazer quedas no faturamento se o nível continuar baixo num determinado [10].

Os sistemas logísticos são constituídos de fluxos de dados e de materiais, no qual os fluxos de informações movem e controlam os fluxos de materiais [11]. Logo, analisar ambos os fluxos desde o inicio do processo em que o consumidor decide considerar a efetuação do pedido até o ultimo instante em que recebe e realiza o pagamento é a melhor forma de entender e controlar o ciclo de pedido e o sistema de processamento de pedidos.

Visando isso um dos maiores problemas para as organizações é ter prejuízos com estoques parados, pois geram custos, manutenção e podem ter avarias, isso prejudica o desempenho que as empresas necessitam apresentar de otimização, o qual as operações de suprimento são fundamentais ao fluxo da Cadeia Logística, em todas suas etapas, para terem 
competitividade no mercado, assim surge a questão que norteou o presente estudo: como a aplicação do método LEC - Lote Econômico Compras pode auxiliar a minimização de custos?

O objetivo geral desta pesquisa é executar uma revisão da literatura referente ao lote econômico de compra - LEC com a intenção de verificar se o mesmo melhora as condições de custos, influenciando um melhor desempenho gerencial e levando a eficiência na capacidade da organização.

No presente trabalho, é apresentado inicialmente o referencial teórico sobre a importância do estudo e pesquisa referente à utilização do lote econômico de compra. Logo, descreve-se a metodologia adotada. Após é aplicada a análise bibliométrica, com a apresentação da coleta de dados e resultados. Por último as considerações finais do estudo são demonstradas, no qual revela os temas que surgem da análise da produção literária e esses podem servir para pesquisas futuras.

\section{REFERENCIAL TEÓRICO}

Nos dias atuais um fator que está em alta é a competitividade nos mercados empresariais, o que leva as organizações a procurarem alternativas as quais possam utilizar como vantagem diante dos seus concorrentes. Os estoques têm grande parcela de utilização no investimento de capital, portanto, precisam ser vistos com supra importância a qual representam para progresso dos negócios e, por conseguinte os lucros. Isto posto, reconhecer a otimização de estoques tem que ser um dos principais foco e objetivo da empresa a ser conquistado pelos gestores. Neste cenário, o controle de estoque é de máxima relevância para o avanço porque age na administração dos produtos em questão de sua necessidade e disponibilidades no armazém durante toda a produção [19].

Os setores de finanças têm como principal preocupação manter um estoque reduzido no máximo, com objetivo de evitar desperdício já o setor de venda e manufatura prefere um estoque mais amplo com a capacidade para se precaver de faltas inesperadas, assim consegue-se representar a necessidade da importância de se estudar o estoque para avaliar e tomar as melhores decisões [4].

$\mathrm{Na}$ indústria e no comércio é empregado o controle do estoque como base para registrar, supervisionar e conduzir os movimentos das mercadorias e produtos por todo o processo da produção até a finalização da venda, o estoque é composto pelas matériasprimas, produtos em fabricação, finalizados e os já expostos nos comércios, material de embalagens e aplicação e peças nas indústrias [1].

Uma das mais essenciais ferramentas de logística que foram criadas e um dos mais excelentes instrumentos utilizados para a definição de medida exata a ser adquirida de cada item para o estoque é o Lote Econômico de Compra [7].

O controle Lote Econômico de Compras (LEC) tem como característica encontrar a quantidade exata a ser produzida ou comprada por pedido a qual os custos sejam os mais reduzidos tanto para o armazenamento quanto para os pedidos, para que se consiga alcançar a maximização do lucro, portanto ele averigua a demanda necessária com previsão, podendo quando for preciso solicitar a encomenda antes do planejado, as organizações que podem empregar este tipo de controle em sua gestão podem ser tanto empresas com perfil de demanda puxada como empurrada [5].

O modelo de controle Lote Econômico de compras (LEC) foi elaborado em 1913 por Ford Harris com fundamento numa quantidade especifica que corresponde à ótima, sendo esta a com o menor custo de estoque e pedido que engloba a preparação do produto, transporte e pedido [6]. Este método pode analisar que quanto maior a quantidade de produção mais reduzido fica o custo devido ao ganho da proporção. 
A função do controle LEC é definir de várias estratégias possíveis qual é a melhor entre elas que seria precisar a quantia que deve ser mantida em depósito e qual melhor período para ser feito novos pedidos com o objetivo de minimização de custos de todo o processo de produção encontrando a quantidade ótima para realizar pedidos [17].

Os principais pressupostos da formulação clássica do LEC são [21]:

a) A demanda é determinística, constante e contínua;

b) O lead time de ressuprimento é determinístico e constante;

c) Faltas de produtos e backorders (entregas com atraso) não são permitidas;

d) Custos de pedido e de estoque são independentes do tamanho da ordem (não existem, por exemplo, descontos por quantidade) e não variam no tempo;

e) Pedido chega completo em um único instante de tempo;

f) Itens diversos são pedidos de forma independente, ou seja, não são consideradas possibilidades de um pedido com vários itens;

g) Não existem restrições, como espaço de armazenamento e capacidade de transporte.

Estipulam-se os custos de falta ou custos de falhas por intermédio de lucros cessantes pela falta do fornecimento. Essas perdas de lucros prejudica a organização quando consumidores cancelam seus pedidos, quando custos adicionais que são necessários para resolver algo que não estava previsto na produção, podendo ser causados por ter havido substituições com matéria prima de terceiros, pelo não cumprimento de prazos ou pela visão que os clientes têm sobre a empresa, que no caso de desapontamento de não conseguir o produto no momento e como almejava acaba optando por comprar num concorrente e assim o beneficiando como consequência [3].

\section{METODOLOGIA}

No que tange o propósito da pesquisa é conseguir alcançar as características de algo sobre alguma população em especifica [16]. Isto posto, no referente estudo a população fazse os artigos publicados os quais compõem o portfólio de 18 artigos alinhados a pesquisa, os mesmos selecionados através das buscas nas bases de dados online que foram Proquest, Science Direct, Web Of Science e Scopus.

Quando o projeto busca mensurar relações entre variáveis é indicada a utilização da pesquisa quantitativa, neste tipo de pesquisa a coleta é feita anterior a análise, na qual os dados coletados são analisados estatisticamente, visto, este trabalho é de caráter quantitativo, pois se realizou a pesquisa bibliográfica, para a verificação associativa da pesquisa, que engloba toda bibliografia já tornada pública em relação ao tema de estudo, como publicações avulsas, boletins, jornais, revistas, livros, pesquisas, monografias entre outros meios [15; 16$]$.

A pesquisa descritiva não procura a explicação de algo e sim o descreve diante dos dados e resultados coletados, não mostra a resposta de porquê alguma definição como resultado, porém associa as decorrências da pesquisa. Portanto esse estudo se enquadra de caráter descritivo, pois através das informações que foram coletadas pela bibliometria, descreveu-se e relacionou-se as mesmas. Quando a intenção de um projeto é explorar um tema que foi pouco estudado, a pesquisa exploratória é a indicada [16]. Logo, definisse com delineamento exploratório esta pesquisa.

\section{ANÁLISE BIBLIOMÉTRICA}

A bibliometria consiste em uma técnica quantitativa e estatística de medição dos índices de produção e disseminação do conhecimento científico, contribuindo para tomadas de decisão na gestão da informação e do conhecimento, uma vez que auxilia na organização e sistematização de informações científicas e tecnológicas [2; 8]. 
A análise bibliométrica é utilizada em diversas áreas do conhecimento como metodologia para a obtenção de indicadores de avaliação da produção científica. De forma geral, o princípio da bibliometria é analisar a atividade científica ou técnica, pelo estudo quantitativo das publicações e o seu principal objetivo é o desenvolvimento de indicadores cada vez mais confiáveis [18].

Para a realização desta análise dividiu-se o processo em duas fases, a primeira consiste na coleta de dados através de bases de dados e na segunda fase são apresentados os resultados provenientes da análise.

\subsection{COLETA DE DADOS}

A escolha das bases de dados online foi a primeira etapa para criação do Portfólio Bibliográfico, sendo escolhidas as seguintes: Proquest, Science Direct, Web of Science, Scopus. Para realizar a pesquisa nestas bases foram utilizadas as seguintes combinações de palavras-chave: "economic order quantity" e cost structures, "economic order quantity" e lot-sizing, "economic order quantity" e optimization of the order quantity, "economic order quantity" e order management, eoq e cost structures, eoq e lot-sizing, eoq e optimization of the order quantity, eoq e order management, "inventory control" e cost structures, "inventory control" e lot-sizing, "inventory control" e optimization of the order quantity, "inventory control" e order management.

Foram realizadas doze pesquisas em cada base de dados, as quais totalizaram 19.372 documentos, após a utilização do filtro de livros, seminários e duplicados foram deletados 12.030 documentos ficando 7.342 documentos os quais com a leitura dos títulos foram excluídos 6.927 gerando 415 documentos, posterior a leitura destes foram excluídos os que eram alinhados a marketing, Just in time, deterioração, itens imperfeitos e os que tratavam da etapa de fabricação, concluiu-se em 24 artigos alinhados a pesquisa, destes, seis não possuem texto na íntegra disponível, conforme o Quadro 1 a seguir.

\begin{tabular}{|cc|}
\multicolumn{2}{c}{ Quadro 1 - Total de documentos pesquisados } \\
\hline Total sem filtro & 19.372 \\
\hline Total com filtro (livros, seminários e duplicados) & 7.342 \\
Total com filtro (títulos) & 415 \\
Total com filtro (resumos) & 24 \\
Total do portfólio & 18 \\
\hline
\end{tabular}

Fonte: Dados da pesquisa

Na base de dados Proquest a busca com as 12 combinações, sem filtro totalizou 11.198 documentos, após o filtro (exclusão de livros, seminários e duplicados) totalizaram 3.797 documentos, a partir destes foi realizada a leitura e finalizou com 11 artigos alinhados a pesquisa como demonstrado no Quadro 2.

Quadro 2 - Pesquisa na base Proquest

\begin{tabular}{|cccc|}
\hline Combinações & $\begin{array}{c}\text { Resultado } \\
\text { s sem } \\
\text { filtro }\end{array}$ & $\begin{array}{c}\text { Resultados } \\
\text { com filtro }\end{array}$ & $\begin{array}{c}\text { Alinhados } \\
\text { a pesquisa }\end{array}$ \\
\hline $\begin{array}{c}\text { "economic order quantity" e cost structures } \\
\text { "economic order quantity" e lot-sizing }\end{array}$ & 498 & 423 & 1 \\
"economic order quantity" e optimization of the order \\
$\begin{array}{c}\text { quantity } \\
\text { "economic order quantity" e order management }\end{array}$ & 887 & 297 & 0 \\
\hline
\end{tabular}




\begin{tabular}{|cccc|} 
eoq e cost structures & 676 & 276 & 0 \\
eoq e lot-sizing & 518 & 62 & 0 \\
eoq e optimization of the order quantity & 991 & 77 & 1 \\
eoq e order management & 1.861 & 203 & 1 \\
"inventory control" e cost structures & 165 & 95 & 0 \\
"inventory control" e lot-sizing & 802 & 323 & 0 \\
"inventory control" e optimization of the order & 2028 & 943 & 0 \\
quantity & 569 & 515 & 0 \\
\hline "inventory control" e order management & 11.198 & 3.797 & 11 \\
\hline Total
\end{tabular}

Fonte: Dados de Pesquisa

Na base de dados Science Direct a busca com 12 combinações, sem filtro totalizou 811 documentos, após o filtro (exclusão de livros, seminários e duplicados) totalizaram 517 documentos, a partir destes foi realizada a leitura e finalizou com dois artigos alinhados a pesquisa como demonstrado no Quadro 3.

Quadro 3 - Pesquisa na base Science Direct

\begin{tabular}{|cccc|}
\hline Combinações & $\begin{array}{c}\text { Resultados } \\
\text { sem filtro }\end{array}$ & $\begin{array}{c}\text { Resultados } \\
\text { com filtro }\end{array}$ & $\begin{array}{c}\text { Alinhados } \\
\text { a pesquisa }\end{array}$ \\
\hline $\begin{array}{c}\text { "economic order quantity" e cost structures } \\
\text { "economic order quantity" e lot-sizing }\end{array}$ & 12 & 10 & 0 \\
"economic order quantity" e optimization of the order & 80 & 69 & 0 \\
quantity & 55 & 22 & 1 \\
"economic order quantity" e order management & 75 & 53 & 1 \\
eoq e cost structures & 24 & 5 & 0 \\
eoq e lot-sizing & 114 & 26 & 0 \\
eoq e order management & 35 & 1 & 0 \\
"inventory control" e cost structures & 71 & 69 & 0 \\
"inventory control" e lot-sizing & 69 & 64 & 0 \\
"inventory control" e optimization of the order & 89 & 68 & 0 \\
quantity & 37 & 15 & 0 \\
"inventory control" e order management & 150 & 115 & 0 \\
\hline Total & 811 & 517 & 2 \\
\hline
\end{tabular}

Fonte: Dados de Pesquisa

Na base de dados Web of Science a busca com as 12 combinações, sem filtro totalizou 3.395 documentos, após o filtro (exclusão de livros, seminários e duplicados) totalizaram 1.356 documentos, a partir destes foi realizada a leitura e finalizou com três artigos alinhados a pesquisa como demonstrado no Quadro 4.

Quadro 4 - Pesquisa na base Web of Science

\begin{tabular}{|cccc|}
\hline Combinações & $\begin{array}{c}\text { Resultados } \\
\text { sem filtro }\end{array}$ & $\begin{array}{c}\text { Resultados } \\
\text { com filtro }\end{array}$ & $\begin{array}{c}\text { Alinhados } \\
\text { a pesquisa }\end{array}$ \\
\hline "economic order quantity" e cost structures & 21 & 11 & 0 \\
"economic order quantity" e lot-sizing & 285 & 158 & 2 \\
"economic order quantity" e optimization of the order & 157 & 29 & 0
\end{tabular}




\begin{tabular}{|c|c|c|c|}
\hline quantity & & & \\
\hline "economic order quantity" e order management & 229 & 74 & 1 \\
\hline eoq e cost structures & 50 & 12 & 0 \\
\hline eoq e lot-sizing & 534 & 222 & 0 \\
\hline eoq e optimization of the order quantity & 149 & 14 & 0 \\
\hline eoq e order management & 269 & 49 & 0 \\
\hline "inventory control" e cost structures & 436 & 236 & 0 \\
\hline "inventory control" e lot-sizing & 506 & 226 & 0 \\
\hline "inventory control" e optimization of the order quantity & 123 & 25 & 0 \\
\hline "inventory control" e order management & 636 & 300 & 0 \\
\hline Total & 3.395 & 1.356 & 3 \\
\hline
\end{tabular}

Fonte: Dados de Pesquisa

Na base de dados Scopus a busca com as 12 combinações, sem filtro totalizou 3.968 documentos, após o filtro (exclusão de livros, seminários e duplicados) totalizaram 1.672 documentos, a partir destes foi realizada a leitura e finalizou com dois artigos alinhados a pesquisa como demonstrado no Quadro 5.

Quadro 5 - Pesquisa na base Scopus

\begin{tabular}{|cccc|}
\hline Combinações & $\begin{array}{c}\text { Resultados } \\
\text { sem filtro }\end{array}$ & $\begin{array}{c}\text { Resultados } \\
\text { com filtro }\end{array}$ & $\begin{array}{c}\text { Alinhados } \\
\text { a pesquisa }\end{array}$ \\
\hline $\begin{array}{c}\text { "economic order quantity" e cost structures } \\
\text { "economic order quantity" e lot-sizing }\end{array}$ & 17 & 15 & 0 \\
"economic order quantity" e optimization of the order & 344 & 74 & 0 \\
quantity & 449 & 179 & 1 \\
"economic order quantity" e order management & 49 & 18 & 0 \\
eoq e cost structures & 153 & 41 & 0 \\
eoq e lot-sizing & 243 & 13 & 0 \\
eoq e optimization of the order quantity & 354 & 28 & 0 \\
eoq e order management & 755 & 489 & 0 \\
"inventory control" e cost structures & 806 & 469 & 0 \\
"inventory control" e lot-sizing & 576 & 203 & 0 \\
"inventory control" e optimization of the order & 52 & 34 & 0 \\
\hline "inventory control" e order management & 3.968 & 1.672 & 2 \\
\hline Total & & & \\
\hline
\end{tabular}

Fonte: Dados de Pesquisa

A representação percentual de artigos alinhados a pesquisa por cada base de dados foi de $61 \%$ na base Proquest, $17 \%$ na base Web of Science, $11 \%$ na base Scopus e $11 \%$ na base Science Direct, como demonstrada na Fig. 1 a seguir. As bases que apresentaram menor porcentagem foi Science Direct e Scopus com apenas dois artigos cada uma delas e a base que apresentou a maior porcentagem foi Proquest com 11 artigos.

Figura 1 - Representação Percentual por Bases de Dados 


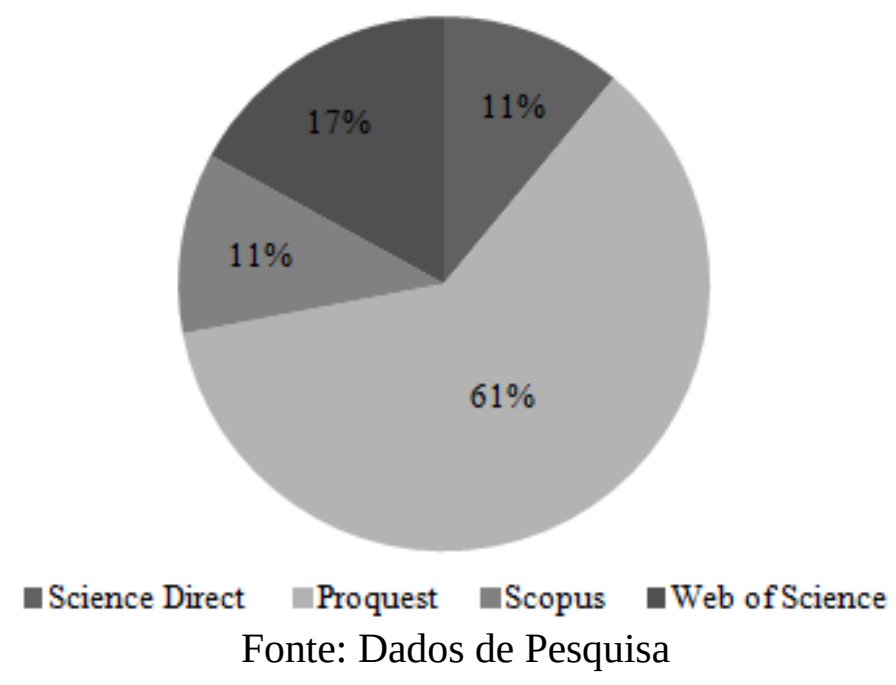

\subsection{RESULTADOS}

Nesta etapa serão demonstrados os dados resultantes das análises de produção e autoria, conteúdos, citações dos artigos e estudo das referências bibliográficas, os quais foram apresentados a partir da bibliometria.

\subsubsection{Estudo de Produção e Autoria}

No que tange o estudo de produção e autoria dos artigos presentes no portfólio bibliométrico, fez-se a análise da frequência das publicações por ano. A Fig. 2 demonstra no gráfico a distribuição do portfólio, variando do ano de 1989 até 2017. O ano 2013 teve destaque com maior número de publicações, representando 22,2\% com quatro artigos, seguido dos anos de 2007 e 2017 que representam 11,1\% cada com dois artigos e os demais anos representam 5,5\% com um artigo cada.

Figura 2 - Publicações dos Artigos

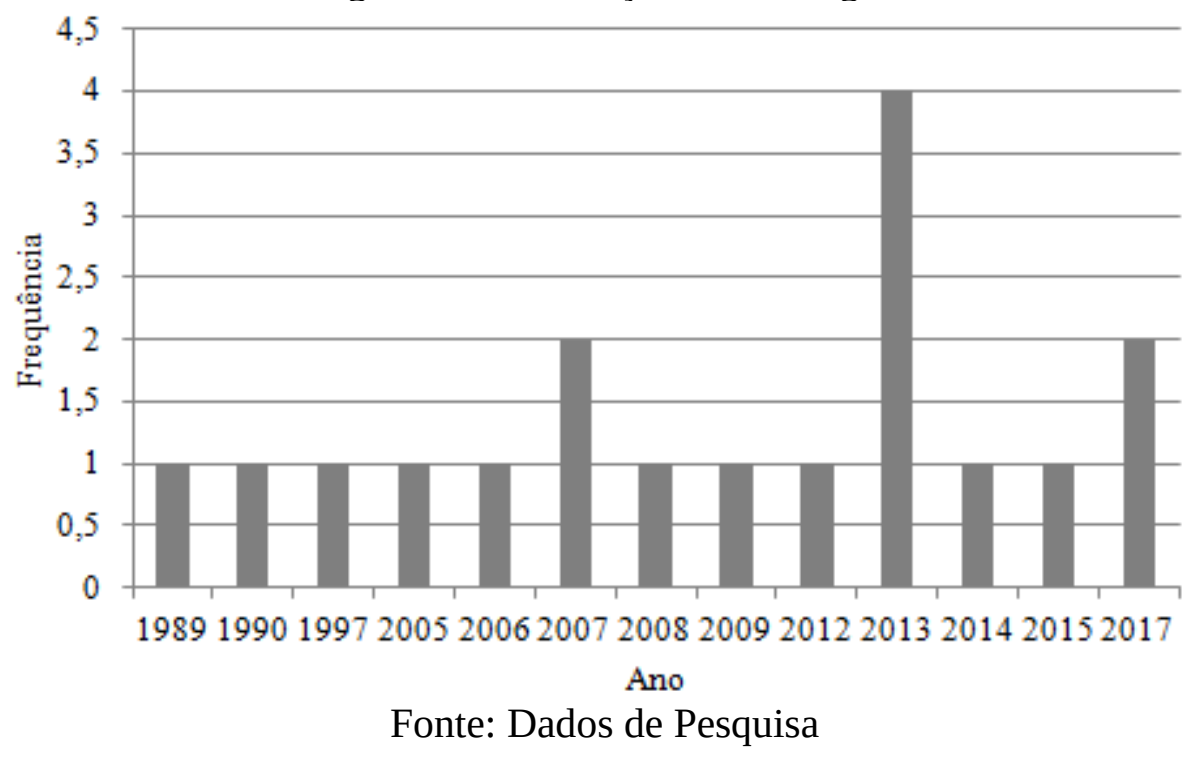

Ao analisar as participações mais relevantes dos autores nos 18 artigos, pode-se observar, como é demonstrado no Quadro 6, que os autores David Pentico, Ata Allah Taleizadeh e Huang Yung-Fu, são os que aparecem em mais publicações, assim 
representando 7,14\% cada com três artigos nesta amostra, os demais apenas com um artigo ficando com $2,38 \%$ cada.

Quadro 6 - Autores

\begin{tabular}{|ccc|}
\hline Autores & Participações & $\%$ \\
\hline Pentico, D. W. & 3 & 7,14 \\
Taleizadeh, A. A. & 3 & 7,14 \\
Yung-Fu, H. & 3 & 7,14 \\
\hline
\end{tabular}

Fonte: Dados de Pesquisa

Ao analisar as universidades que mais produziram no portfólio, verificou-se 14 universidades, das quais três universidades apresentaram maior representatividade, sendo Chaoyang University of Technology com 16,67\%, American University of Beirut com $11,11 \%$, University of Tehran com 11,11\% e as demais universidades, representando $61,16 \%$ do portfólio, apenas com um artigo cada, como demonstrado no Quadro 7.

Quadro 7 - Universidades com maior número de participações

\begin{tabular}{|ccc|}
\hline Universidades dos artigos publicados & $\begin{array}{c}\text { Número de } \\
\text { artigos }\end{array}$ & $\%$ \\
\hline Chaoyang University of Technology & 3 & 16,67 \\
American University of Beirut & 2 & 11,11 \\
University of Tehran & 2 & 11,11 \\
Auburn University & 1 & 5,56 \\
Vidyasagar University & 1 & 5,56 \\
Iran University of Science and Technology & 1 & 5,56 \\
PSNA College of Engineering and Technology & 1 & 5,56 \\
Southern Methodist University & 1 & 5,56 \\
Tamkang University & 1 & 5,56 \\
Universidad de Valladolid & 1 & 5,56 \\
Universidade Federal Fluminse & 1 & 5,56 \\
Universitat Internacional de Catalunya & 1 & 5,56 \\
University of Sheffield & 1 & 5,56 \\
University Park & 1 & 5,56 \\
\hline
\end{tabular}

Fonte: Dados da pesquisa

Ao analisar as revistas das publicações, notou-se que, das 15 revistas analisadas, as revistas Applied Mathematical Modelling, International Journal of Production Economics e The Journal of the Operational Research Society apresentaram o maior número de publicações, representando 33,33\% com dois artigos cada, as demais representaram 66,72\% do portfólio, com apenas um artigo cada (Quadro 8).

Quadro 8 - Revistas

\begin{tabular}{|ccc|}
\hline Revista & Frequência & $\%$ \\
\hline Applied Mathematical Modelling & 2 & 11,11 \\
International Journal of Production Economics & 2 & 11,11 \\
The Journal of the Operational Research Society & 2 & 11,11 \\
An International Journal of Optimization and Control & 1 & 5,56
\end{tabular}




\begin{tabular}{|ccc|}
\hline Asia - Pacific Journal of Operational Research & 1 & 5,56 \\
Computers \& Industrial Engineering & 1 & 5,56 \\
European Journal of Operational Research & 1 & 5,56 \\
IIE Annual Conference. Proceedings & 1 & 5,56 \\
International Journal for Engineering Modelling & 1 & 5,56 \\
International Journal of Systems Science & 1 & 5,56 \\
Journal of Management Analytics & 1 & 5,56 \\
Human Factors and Ergonomics in Manufacturing \& Service & 1 & 5,56 \\
Industries & 1 & 5,56 \\
IIE Annual Conference. Proceedings & 1 & 5,56 \\
Journal of Information \& Optimization Sciences & 1 & 5,56 \\
\hline
\end{tabular}

Fonte: Dados da pesquisa

No Quadro 9 está demonstrado a análise dos países de origem dos artigos que compõem o portfólio. Nesta análise considerou-se somente o país de origem do primeiro autor do estudo, encontrando um total de oito países. Os países que mais publicaram artigos em relação ao tema abordado, foram a China e Estados Unidos, representando 44,44\% do portfólio com quatro artigos cada, os demais seis países representaram 44,44\% e 11,12\%, com, respectivamente, dois e um artigos.

Quadro 9 - Países com maior número de participações

\begin{tabular}{|ccc|}
\hline Países & Número de artigos & $\%$ \\
\hline China & 4 & 22,22 \\
USA & 4 & 22,22 \\
Espanha & 2 & 11,11 \\
Índia & 2 & 11,11 \\
Irã & 2 & 11,11 \\
Líbano & 2 & 11,11 \\
Brasil & 1 & 5,56 \\
Inglaterra & 1 & 5,56 \\
\hline
\end{tabular}

Fonte: Dados da pesquisa

\subsubsection{Análise dos Conteúdos}

Nesta etapa foi analisado as palavras-chave dos artigos, verificou-se quais as mais utilizadas pelos autores, onde observou-se que do total de 73 palavras a com maior frequência foi Inventory sendo mencionada em nove artigos, seguida de EOQ em sete, Partial backordering em quatro, trade credit em três, com duas citações cash discount e Full backordering e as demais 46 palavras-chave foram citadas uma única vez. A distribuição das palavras-chave pode ser visualizada na Fig. 3.

Figura 3 - Palavras-chave com maior número de participações 


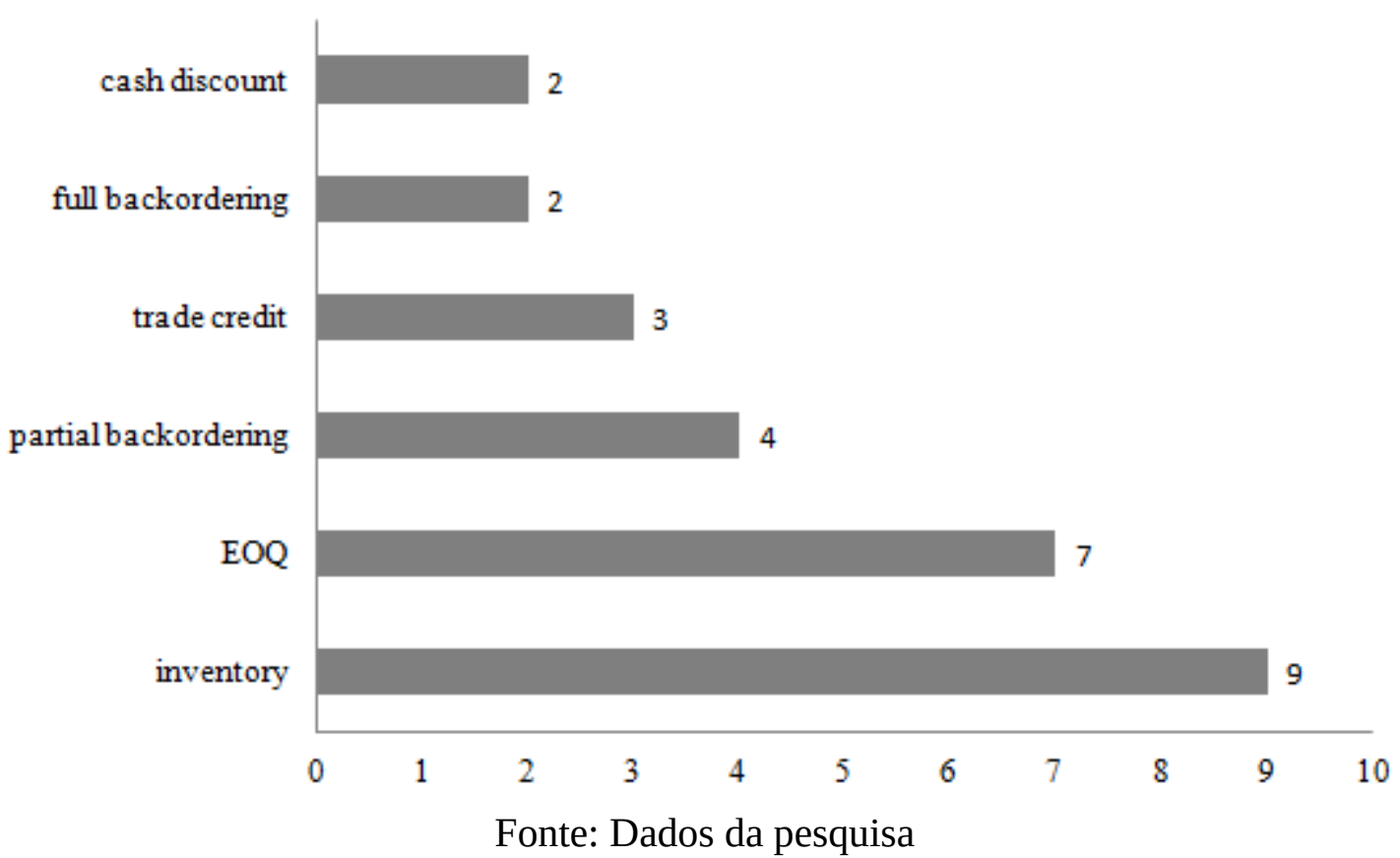

Na segunda análise desta etapa, pôde-se averiguar a quantidade de autores por cada artigo dos 18 alinhados, a maior frequência constatada foi de 50\% com dois autores em nove artigos, na sequência 22,22\% dos artigos, ou seja, quatro, escrito por três autores, 16,67\% equivalente a três artigos escritos por um autor e $11,11 \%$ corresponde a dois artigos escritos por quatro autores como expõe o Quadro 10.

Quadro 10 - Número de autores por artigo

\begin{tabular}{|ccc|}
\hline Número de autores & Número de artigos & $\%$ \\
\hline 2 autores & 9 & 50,00 \\
3 autores & 4 & 22,22 \\
1 autor & 3 & 16,67 \\
4 autores & 2 & 11,11 \\
\hline
\end{tabular}

Fonte: Dados da pesquisa

\subsubsection{Análise de Citação dos Artigos}

Esta etapa do estudo teve como objetivo identificar a relevância dos artigos presentes no portfólio bibliográfico, através da análise de citação dos mesmos. Através do Google Acadêmico, no período de Agosto de 2019, investigou-se o número de citação de cada artigo, dando destaque, com 65 citações, o artigo An economic order quantity model with partial backordering and a special sale price, e três artigos não apresentaram nenhuma citação que são An EOQ Binary Model with All Units Discounts for Multiproducts, An EOQ model under supplier trade credit policy depending on the order quantity e An EOQ model under trade credit and conditional cash discount. A distribuição das citações dos artigos é mostrada no Quadro 11.

Quadro 11 - Artigos mais citados

\begin{tabular}{|lc|}
\hline \multicolumn{1}{|c|}{ Título } & Citação \\
\hline $\begin{array}{l}\text { An economic order quantity model with partial backordering and a special sale } \\
\text { price }\end{array}$ & 65 \\
\hline
\end{tabular}




\begin{tabular}{|lc|}
$\begin{array}{l}\text { An Economic Order Quantity model with partial backordering and all-units } \\
\text { discount }\end{array}$ & 51 \\
EOQ Under Date-Terms Supplier Credit & 37 \\
$\begin{array}{l}\text { A general model for EOQ inventory systems with partial backlogging and linear } \\
\text { shortage costs }\end{array}$ & 34 \\
$\begin{array}{l}\text { An economic order quantity model with partial backordering and incremental } \\
\text { discount }\end{array}$ & 28 \\
$\begin{array}{l}\text { EOQ Systems: The Case of an Increase in Purchase Cost } \\
\text { Time-limited free back-orders EOQ model }\end{array}$ & 27 \\
Na EOQ model with limited storage capacity under trade credits & 25 \\
$\begin{array}{l}\text { An algorithm for solution of an interval valued EOQ model } \\
\text { EOQ revisited: The case of unequal and integral order quantities }\end{array}$ & 22 \\
$\begin{array}{l}\text { EOQ holds under stochastic demand, a technical note } \\
\text { EOQ-based Inventory Control Policies for Perishable Items: The Case of Blood } \\
\text { Plasma Inventory Management }\end{array}$ & 12 \\
$\begin{array}{l}\text { Analysis and optimization of an EOQ inventory model with promotional efforts } \\
\text { and back ordering under delay in payments }\end{array}$ & 11 \\
$\begin{array}{l}\text { EOQ Model: The Case in Which the Placing of Orders Is Rewarded } \\
\begin{array}{l}\text { Economic order quantity under retailer partial trade credit in two-echelon } \\
\text { supply chain }\end{array}\end{array}$ \\
\hline
\end{tabular}

Fonte: Dados da pesquisa

\subsubsection{Estudo das Referências Bibliográficas}

O objetivo dessa etapa foi identificar entre os 18 artigos selecionados, quais referências foram mais utilizadas nos artigos do portfólio. Para isso, foram analisadas todas as 342 referências bibliográficas das obras, para melhor representação no Quadro 12, tomouse como ponto de corte o número de quatro citações por obra. A pesquisa mais utilizada foi Factory, The Magazine of Management com seis citações, com cinco citações foi Inventory Systems e com quatro detectou-se Analysis of Inventory SysIems, An inventory model with deteriorating items under inflation when a delay in payment is permissible, Inventory models with a mixture of backorders and lost sales, The Deterministic EOQ with Partial Backordering: A New Approach, Inventory Management and Production Planning and Scheduling, On the economic order quantity under conditions of permissible delay in payments. Também se teve sete artigos que foram citados três vezes e dez artigos duas vezes, os demais foram utilizados uma única vez.

Quadro 12 - Artigos mais citados nas referências

\begin{tabular}{|cccc|}
\hline Autores & Título & Ano & Citações \\
\hline Harris, F. W. & Factory, The Magazine of Management. & 1913 & 6 \\
Naddor, E. & Inventory Systems & 1966 & 5 \\
Hadley, G. e Whitin, T. M. & Analysis of Inventory SysIems. & 1963 & 4 \\
\hline $\begin{array}{c}\text { Liao, H. C.; Tsai, C. H. e } \\
\text { Su, C. T. }\end{array}$ & $\begin{array}{c}\text { An inventory model with deteriorating } \\
\text { items under inflation when a delay in } \\
\text { payment is permissible. }\end{array}$ & 2000 & 4 \\
$\begin{array}{c}\text { Montgomery, D. C.; } \\
\text { Bazaraa, M. S. e } \\
\text { Keswani, A. K. }\end{array}$ & $\begin{array}{c}\text { Inventory models with a mixture of } \\
\text { backorders and lost sales. }\end{array}$ & 1973 & 4 \\
$\begin{array}{c}\text { Pentico, D. W. e } \\
\text { The Deterministic EOQ with Partial }\end{array}$ & 2009 & 4
\end{tabular}




\begin{tabular}{|ccc|c|}
\hline Drake, M. J. & Backordering: A New Approach & & \\
Silver, E. A.; Pyke, D. F. e & $\begin{array}{c}\text { Inventory Management and Production } \\
\text { Peterson, R. }\end{array}$ & 1998 & 4 \\
Planning and Scheduling. & $\begin{array}{c}\text { On the economic order quantity under } \\
\text { conditions of permissible delay in } \\
\text { payments. }\end{array}$ & 2002 & 4 \\
\hline
\end{tabular}

Fonte: Dados da pesquisa

\section{CONSIDERAÇÕES FINAIS}

Este estudo teve como objetivo executar uma revisão da literatura referente ao lote econômico de compra - LEC, com a intenção de verificar se o mesmo melhora as condições de custos, influenciando um melhor desempenho gerencial e levando a eficiência na capacidade da organização, para tal foi realizada uma análise bibliográfica nas bases de dados online.

Ao analisar os resultados da analise bibliométrica constata-se que entre os artigos encontrados os autores que mais participaram da amostra foram David Pentico, Ata Allah Taleizadeh e Huang Yung-Fu todos com três artigos cada representando 7,14\%. O ano que mais teve publicações foi 2013, demonstrando um momento de grande repercussão do tema. Nas universidades a que teve maior representação foi Chaoyang University of Technology, localizada em Taiwan, com três artigos e logo American University of Beirut, no Líbano e University of Tehran, no Irã, cada com dois artigos. Já nos países destacaram-se China e EUA cada com quatro artigos, assim representando 44,44\%. A palavra-chave mais utilizada foi Inventory por nove vezes e EOQ após por sete vezes. Para análise de quantidade de autores por título a metade do portfólio teve dois autores. O artigo com mais citações foi An economic order quantity model with partial backordering and a special sale price e a referência mais utilizada Factory, The Magazine of Management.

Como limitação de pesquisa destaca-se a indisponibilidade de todos artigos online, e mesmo realizando-se as buscas por meio de palavras-chave, muitos dos documentos não se alinhavam ao projeto em questão. Por fim, o tema de pesquisa analisado no presente estudo, pode ser desenvolvido em pesquisas futuras, buscando abranger um número maior de bases de dados.

\section{REFERÊNCIAS BIBLIOGRÁFICAS}

[1] ANDRADE, L. F. de; OLIVEIRA, I. P. de. Controle de Estoque. Revista Eletrônica Faculdade Montes Belos, v. 4, n. 2, Nov, 2011.

[2] ARAÚJO, C. A. Bibliometria: evolução histórica e questões atuais. Em Questão, v. 12, n. 1, p. 11-32, 2006.

[3] AURÉLIO, M. P. D. Princípios, Conceitos e Gestão. 5. ed. São Paulo: Editora Atlas. 2005

[4] BERTAGLIA, P. R. Logística e gerenciamento da cadeia de suprimento de abastecimento. 2. ed. São Paulo: Saraiva, 2005.

[5] CAMPOS, P. R.; MARTINS, G. M. Administração de materiais e recursos patrimoniais. 1. ed. São Paulo: Saraiva, 2000.

[6] CASTRO, R. L. Planejamento e controle da produção e estoques: um survey com fornecedores da cadeia automobilística brasileira. $111 \mathrm{f}$. Dissertação (Mestrado em Engenharia de Produção), p. 34, São Paulo: POLI/USP, 2005. 
[7] GITMAN, L. J. Princípios de Administração Financeira. São Paulo: HARBRA ltda, 2002.

[8] GUEDES, V. L. S.; BORSCHIVER, S. Bibliometria: uma ferramenta estatística para a gestão da informação e do conhecimento, em sistemas de informação, de comunicação e de avaliação científica e tecnológica. CINFORM- Encontro Nacional de Ciência da Informação, v. 6, 2005.

[9] IETEC - Instituto de Educação e Tecnologia. Gestão de estoques na cadeia de suprimentos, 2017. Disponível em: <http://www.ietec.com.br/imprensa/gestao-deestoques-na-cadeia-de-suprimentos/>. Acesso em: 30 jun. 2018.

[10] ILOS - Especialistas em Logística e Supply Chain. Gestão de Estoques, 2017. Disponível em: <http://www.ilos.com.br/web/solucoes-por-tema/gestao-de-estoques/> Acesso em: 30 jun. 2018.

[11] ILOS - Especialistas em Logística e Supply Chain. O impacto da gestão de estoques nos custos e no nível de serviço, 2017. Disponível em:

$<$ http://www.ilos.com.br/web/o-impacto-da-gestao-de-estoques-nos-custos-e-no-nivelde-servico/> Acesso em: 30 jun. 2018.

[12] ILOS - Especialistas em Logística e Supply Chain. O sistema de processamento de pedidos e a gestão do ciclo do pedido, 2003. Disponível em: <http://www.ilos.com.br/ web/o-sistema-de-processamento-de-pedidos-e-a-gestao-do-ciclo-do-pedido/>. Acesso em: 30 jun. 2018.

[13] Instituto Empreender Endeavor Brasil. Gestão de custos: como ter um bom controle financeiro, 2015. Disponível em: <https://endeavor.org.br/financas/gestao-de-custos/>. Acesso em: 30 jun. 2018.

[14] Intelipost. Como usar a gestão de estoque como ferramenta para redução de custos do negócio, 2017. Disponível em: <https://www.intelipost.com.br/blog/como-usar-agestao-de-estoque-como-ferramenta-para-reducao-de-custos-do-negocio/>. Acesso em: 30 jun. 2018.

[15] LAKATOS, E. M.; MARCONI, M. A. Fundamentos de metodologia científica. $5^{\mathrm{a}}$ ed. São Paulo: Atlas, 2003.

[16] ROESCH, S. M. A. Projetos de estágio e de pesquisa em administração: guia para estágios, trabalhos de conclusão, dissertações e estudos de caso. 3. ed. São Paulo: Atlas, 2015.

[17] ROGERS, P.; RIBEIRO, K. C. S.; ROGERS, D. Avaliando o risco na gestão financeira de estoques. In: Simpósio de administração da produção, logística e operações internacionais, 2004. Anais do VII - SIMPOI 2004 - FGV - EAESP.

[18] SANTOS, R. N. Produção científica: por que medir? O que medir? R.D.B.C.I. Campinas, v. 1, n.1, p. 22-38, jul./dez. 2003.

[19] SANTOS, T. S.; COSTA, D. R. M.; ALVES, L. F.; ROCHA, N. S.; SOUZA, S. C. Aplicação de ferramentas da gestão de estoque e custo: um estudo de caso em uma loja de artigos e acessórios para automóveis em Marabá - PA. Revista Latino-Americana de Inovação e Engenharia de Produção, [S. l.], v. 5, n. 8, p. 98-116, 2017.

[20] SEBRAE - Serviço de Apoio às Micro e Pequenas Empresas. A importância da análise periódica de demanda para a gestão de estoques, 2017. Disponível em: $<$ http://blog.sebrae-sc.com.br/gestao-de-estoque/>. Acesso em: 30 jun. 2018. 
[21] SEVERO FILHO, J. Administração de logística integrada: materiais, PCP e marketing. 2 ed. Rio de Janeiro: E-papers, 2006. 